This is the author's final, peer-reviewed manuscript as accepted for publication. The publisher-formatted version may be available through the publisher's web site or your institution's library.

\title{
Analyses of ITS and LSU gene regions provide congruent results on fungal community responses
}

Shawn P. Brown, Anne R. Rigdon-Huss, Ari Jumpponen

\section{How to cite this manuscript}

If you make reference to this version of the manuscript, use the following information:

Brown, S. P., Rigdon-Huss, A. R., \& Jumpponen, A. (2014). Analyses of ITS and LSU gene regions provide congruent results on fungal community responses. Retrieved from http://krex.ksu.edu

\section{Published Version Information}

Citation: Brown, S. P., Rigdon-Huss, A. R., \& Jumpponen, A. (2014). Analyses of ITS and LSU gene regions provide congruent results on fungal community responses. Fungal Ecology, 9, 65-68.

Copyright: @ 2014 Elsevier Ltd and The British Mycological Society

Digital Object Identifier (DOI): doi:10.1016/j.funeco.2014.02.002

Publisher's Link:

http://www.sciencedirect.com/science/article/pii/S175450481400021X

This item was retrieved from the K-State Research Exchange (K-REx), the institutional repository of Kansas State University. K-REx is available at http://krex.ksu.edu 
Analyses of ITS and LSU gene regions provide congruent results on fungal community responses

Shawn P. Brown ${ }^{1 *}$

Anne R. Rigdon-Huss ${ }^{2}$

Ari Jumpponen ${ }^{1}$

1: Division of Biology, Kansas State University

2: Department of Grain Science and Industry, Kansas State University

Keywords: ITS, LSU, Ecological Analysis, 454-Sequencing

* Corresponding Author

Shawn P. Brown

Division of Biology

116 Ackert Hall

Kansas State University

Manhattan, KS 66506

Phone: 785-532-3934

Fax: 785-532-6653 


\begin{abstract}
The Internal Transcribed Spacer (ITS) regions and the Large Subunit (LSU) of the nuclear ribosomal RNA (rRNA) gene complex are commonly used to elucidate questions in fungal community ecology. Here, we compared the congruence across these gene regions using two ecological experiments (primary successional dynamics at a receding glacier forefront and community dynamics in stored Sorghum biomass), in which both ITS1 and LSU were sequenced from the same DNA extracts. We analyzed richness, diversity and evenness estimators along with community shifts inferred from ordination analyses. Our analyses show that ITS and LSU provide similar results and consistent conclusions. Taken together, we conclude that either gene region is appropriate for testing ecological hypotheses as long as there are no a priori hypotheses that preclude the use of one gene region over the other.
\end{abstract}


The Internal Transcribed Spacer (ITS) regions of the rRNA gene complex have been the primary targets for community ecology that relies on sequencing and sequence annotation for taxonomic information (Peay et al, 2008), so that ITS regions have been selected as the barcode for identification of environmental fungal sequences (Schoch et al., 2012). There is little debate on whether ITS is a robust tool in fungal community ecology. ITS is also powerful in identifying fungi at species level taxonomic resolution. However, ITS regions are hyper-variable and great care must be exercised in ITS sequence data analyses to minimize user-generated biases (see Nilsson et al., 2012). Often, novice investigators diving into next-generation sequencing targeting fungi are inclined to attempt global alignment of the ITS data to generate a distance matrix prior to binning sequences into OTUs as is commonly done with bacterial $16 \mathrm{~S}$ sequences. It would seem reasonable to analyze fungal sequence data similarly, but this is usually inappropriate for filtering and clustering fungal ITS sequences because of the region's hyper-variability that precludes global alignments. Instead, ITS sequences can be pairwise aligned to generate a distance matrix for each pair of sequences. For larger datasets, however, this is often computationally expensive. The more conserved small subunit (SSU) or large subunit (LSU) sequences of the rRNA gene complex provide an alternative to ITS for testing ecological hypotheses, but may suffer from limited resolution when resolving obtained taxa. As a result of available reference alignments and the Naïve Bayesian Classifier (Wang et al., 2007), the LSU region in particular has begun to gain some traction (Lothamer et al., 2013; Porras-Alfaro et al., 2014; Weber et al., 2013). This is because these tools expedite analyses and provide access to a curated annotation tool for the sequence data, supporting the integration of evolutionary and ecological questions, similarly to bacterial systems. The available fungus specific primer sets for ITS and LSU regions that permit sequencing environmental fungal communities allow for generating relatively long sequences that can be analyzed using 454-pyrosequencing (Margulies et al., 2005). It is of note that as of yet, there are no verified and minimally biased universal primer pairs that reliably and broadly amplify short enough gene regions within the 
fungal LSU to permit paired-end Illumina or lon Torrent sequencing, although this is an area of current research. As little information is available whether the ITS or LSU provide congruent views of fungal ecology (see Kerekes et al., 2013), we compared two pairs of ITS [primers ITS1f and ITS4] and LSU [primers LROR and LR3; see Amend et al., 2010] sequence data sets generated from the same DNA extracts to evaluate their reliability to describe basic fungal ecology.

We re-analyzed and compared ITS1 and LSU (with the variable region D1) 454-pyrosequencing libraries from two experiments that have been recently published or are currently in various stages of preparation; a total of four 454 sequence libraries. We selected a primary successional system at the forefront of a receding glacier (Brown and Jumpponen, 2014; BioProject PRJNA201483; SRA accessions for individual fastq files: ITS - SRR934164-SRR943234; LSU SRR1016610-SRR1016625, SRR1016734-SRR1016736, SRR1016744SRR1016777, SRR1016785-SRR1016788, SRR1016859-SRR1016865) and an experiment that evaluates effects of storage on fungal communities in stored Sorghum biomass (Rigdon-Huss et al., unpublished; BioProject PRJNA221342; SRA accessions for sff files: ITS - SRR1016401; LSU - SRR1016405). The former evaluates community dynamics over time since deglaciation and as a result of plant establishment, whereas the latter follows fungal communities during six-month storage under various biomass covering treatments. All sequence data from the four libraries were processed identically with MOTHUR (v. 1.31.2; Schloss et al., 2009) according to a modified standard operating protocol (Schloss et al., 2011). Briefly, after denoising the .sff files and truncating all sequences to $250 \mathrm{bp}$ in length, we removed all potentially chimeric reads. The sequence data for each library were pairwise aligned to generate a distance matrix and sequences clustered to OTUs at $97 \%$ sequence similarity using an average neighbor approach. After omission of singleton OTUs, we generated mean diversity and richness estimators based on 1000 iterations at a subsample depth of 200 sequences per experimental unit to minimize biases due to uneven sampling (Gihring et al. 2012): we compared OTU Richness (Sobs), Complement of Simpson's Diversity (1-D) and Simpson's Evenness (ED) using paired t-tests to 
test for dissimilarities between ITS and LSU sequence data sets on an experimental unit basis. As LSU permits global alignment, we also analyzed these data by alignment against a reference (James et al. 2006) in which all nonfungal sequences were removed and the remaining sequences were de novo aligned using MUSCLE. To analyze compositional shifts in the communities, we generated Bray-Curtis distance matrices and visualized communities using Nonmetric Multi-Dimensional Scaling (NMDS) axes $(k=3)$ with loading scores generated based on 1000 iterations for ITS and each of our LSU analysis methods at a 200 sequence subsampling depth. Two-way analysis of variance (ANOVA) was used to test if ecological indices and NMDS axes loading scores change with time, with treatment, or have time $x$ treatment interactions on an experiment-wide basis across the ITS and LSU datasets (see Table S1).

On an experimental unit basis, paired t-tests suggest significant but proportionally minor differences in diversity responses our two experiments between the two rRNA gene regions. However, it is the alignment of the LSU data to a reference that has a greater effect on results than the choice of a target region. The results from our glacier forefront study show that OTU richness, diversity and evenness differ between ITS and the reference aligned LSU data, but not between pairwise-aligned ITS and LSU data (Fig. 1a). This may be due to the relatively high abundance of early diverging taxa and broad taxonomic distribution of fungi present in the samples from the glacier forefront. Most reference alignments, including James' and coauthors' (2006), suffer from sparse sampling of unresolved early diverging clades. The biomass storage study provided a contrasting view. Regardless of how the LSU data were treated, the ITS and LSU data differed consistently in richness, diversity or evenness (Fig.1b). Furthermore, pairwise- and reference-aligned LSU data were similar and consistently deflated in richness, diversity or evenness estimators compared to ITS. Again, this may be due to a phylogenetic distribution as well-characterized Ascomycota dominated the Sorghum biomass communities.

Although the paired t-tests provide interesting comparisons between the ITS and LSU data, experiment-wide ecological responses are usually more pertinent 
for an investigator deciding which gene region to use. In our glacier forefront study, richness, diversity and evenness behaved consistently and were unresponsive to the experimental conditions irrespective of the gene region or how the LSU data were analyzed. Similarly, in the stored biomass study, richness increased storage time across all three analyses. Diversity increased with storage time in LSU data sets (pairwise or reference aligned) with time, but did so only marginally significantly in the ITS data. Interestingly, the ITS data suggested clear treatment differences in evenness, whereas no such response was visible in the LSU data. In sum, experiment-wide, most diversity and richness metrics responded consistently in both systems regardless of the chosen gene region.

Finally, community analyses based on NMDS indicate that ITS and LSU also provide very similar results. Consistently with our original analyses (Brown and Jumpponen, 2014), axis loading scores show trajectories with distance from glacier in ITS and LSU (pairwise and reference aligned) data sets for at least one axis (Fig. 1C), but no effect of the plant establishment. Similarly to the glacier forefront study, the results from the Sorghum storage study are consistent regardless of the gene region or how the LSU data were treated: all analyses congruently suggest shifts in fungal communities over time (Fig. 1d) as well as distinguish one of the four storage methods from others (Table S1).

Overall, we conclude that ITS and LSU regions perform mainly similarly in estimating richness and diversity or in distinguishing treatments in communitywide ordination analyses. There was also congruence in taxa identified between ITS and LSU (see Fig. S1) similarly to analyses by Porras-Alfaro et al. (2014), although obtained taxon affinities depend on inherent primer biases. This suggests that both gene regions are suitable for testing ecological hypotheses and provide comparable results with few exceptions. It is unclear what the underlying reasons for the observed differences are, but may be partly attributable to the variability between the gene regions, distribution of observed taxa, biases resulting from primer selection, or stochastic variability in the library production, sequencing, and analysis steps. Prior to deciding on a target gene 
region to sequence, the investigator must pay heed to the limitations of each gene region and how these may affect the downstream steps in sequencing or analysis. Furthermore, we wish to emphasize that the different sequencing targets may permit testing different hypotheses - a point to bear in mind when designing an experiment that relies on sequencing of variable regions. For example, if OTU identification to the level of species is paramount to the goals of a study, ITS regions are powerful and benefit from the greater number of available sequences in databases. In contrast, hypotheses addressing broad evolutionary ecological processes may be precluded by the inherently unalignable and hyper-variable nature of ITS regions. These caveats aside, we conclude that both ITS or LSU data are suited for analyses fungal community ecology.

\section{Acknowledgements}

This research was partly supported by US National Science Foundation Grant DEB-0516456. The authors' would like to express our great appreciation to Rauni Strömmer, Chad Fox and Laura Fox for assistance with sample collection.

Shawn Brown is supported by the National Science Foundation GK-12 fellowship (NSF DGE-0841414, PI Ferguson). Anne Rigdon-Huss is supported by National Science Foundation IGERT (NSF 0903701 to KSU) I-STAR Bioenergy fellowship and Kansas Alliance for BioRefining and BioEnergy (KABB GAGS92152 to KSU - Grain Science and Industry). 


\section{References}

Amend AS, Seifert KA, Samson R, Bruns TD. 2010. Indoor fungal composition is geographically patterned and more diverse in temperate zones than in the tropics. Proceedings on the National Academy of Sciences 107: 13748-13753.

Brown SP, Jumpponen A. 2014. Contrasting primary successional trajectories of fungi and bacteria in retreating glacier soils. Molecular Ecology 23: 481-197.

Gihring TM, Green AJ, Schadt CW. 2012. Massively parallel rRNA gene sequencing exacerbates the potential for biased community diversity comparisons due to variable library sizes. Environmental Microbiology 14: 285290.

James TY, Kauff F, Schoch CL, Matheny PB, Hofstetter V, et al. 2006. Resonstructing the early evolution of Fungi using a six-gene phylogeny. Nature 443: 818-822.

Kerekes J, Kaspari M, Stevenson B, Nilsson RH, Hartmann M, Amend A, Bruns TD. 2013. Nutrient enrichment increased species richness of leaf litter fungal assemblages in a tropical forest. Molecular Ecology 22: 2827-2838.

Lothamer K, Brown SP, Mattox JD, Jumpponen A. 2013. Comparison of rootassociated communities of native and non-native ectomycorrhizal hosts in an urban landscape. Mycorrhiza (in press) doi: 10.1007/s00572-013-0539-2.

Margulies M, Egholm M, Altman WE, Attiya A, Bader JS et al. 2005. Genome sequencing in microfabricated high-density picolitre reactors. Nature 437: 376380 .

Nilsson RH, Tedersoo L, Abarenkov K, Ryberg M, Kristiansson E, Hartmann M, Schoch CL, Mylander JAA, Bergsten J, Porter TM, Jumpponen A, Vaishampayan 
$\mathrm{P}$, Ovaskainen O, Hallenberg N, Bengtsson-Palme J, Eriksson KM, Larsson KH, Larsson E, Kóljalg U. 2012. Five simple guidelines for establishing basic authenticity and reliability of newly generated fungal ITS sequences. MycoKeys 4: 37-63.

Peay KG, Kennedy PG, Bruns TD. 2008. Fungal community ecology: a hybrid beast with a molecular master. BioScience 58: 799-810.

Porras-Alfaro A, Liu K, Kuske CR, Xie G. 2014. From genus to phylum: largesubunit and internal transcribed spacer rRNA operon regions show similar classification accuracies influenced by database composition. Applied and Environmental Microbiology 80: 829-840.

Schloss PD, Westcott SL, Ryabin T, Hall JR, Hartmann M, Hollister EB, Lesniewski RA, Oakley BB, Parks DH, Robinson CJ, Sahl JW, Stres B, Thallinger GG, Van Horn DJ, Weber CF. 2009. Introducing mother: Open-source, platform-independent, community-suppoeted software for describing and comparing microbial communities. Applied and Environmental Microbiology 75: 7537-7541.

Schloss PD, Gevers D, Westcott SL. 2011. Reducing the effects of PCR amplification and sequencing artifacts on 16S rRNA-based studies. PLOS ONE 6: e27310.

Schoch CL, Seifert KA, Huhndorf S, Robert V, Spouge JL, Levesque CA, Chen W, Fungal Barcoding Consortium. 2012. Nuclear ribosomal internal transcribed spacer (ITS) region as a universal DNA barcode marker for Fungi. Proceedings of the National Academy of Science, USA 109: 6241-6246.

Wang Q, Garrity GM, Tiedje JM, Cole JR. 2007. Naive Bayesian classifier for rapid assignment of rRNA sequences into the new bacterial taxonomy. Applied 
and Environmental Microbiology 73: 5261-5267.

Weber CF, Vilgalys R. Kuske CR. 2013. Changes in fungal community

composition in response to elevated atmospheric $\mathrm{CO}_{2}$ and nitrogen fertilization varies with soil horizon. Frontiers in Microbiology 4: 78 doi:

10.3389/fmicb.2013.00078. 


\section{Figure 1}

Paired t-tests of richness, diversity (1-d) and evenness $\left(E_{D}\right)$ estimators derived from ITS, pairwise aligned LSU, and reference aligned LSU data in the Lyman Glacier Forefront (a) and Stored Sorghum Biomass (b) experiments. Bars indicate mean values across all experimental units and asterisks $\left(^{*}\right)$ represent significant differences at $P \leq 0.05$. Regression analyses of the Non-metric MultiDimensional Scaling (NDMS) loading scores of the first three resolved axes and distance from glacier terminus in the Lyman Glacier Forefront (c) and time of storage in the Stored Sorghum Biomass (d) experiments for ITS and LSU (pairwise and reference aligned). Asterisks indicate significant slopes $(P \leq 0.05)$. Complete test statistics and $P$-values are in Table S1.

\section{Figure S1}

Order level taxonomic affinities for ITS and LSU gene regions for Lyman Glacier forefront and stored Sorghum biomass experiments. Labeled order percentages represent proportion of total OTUs that were classified to order-level for the ten most abundant orders for each experiment. The taxon affinities are largely but not exclusively congruent.

\section{Table S1}

Results from paired t-tests from Lyman Glacier Forefront and Stored Sorghum Biomass experiments comparing diversity estimators derived using ITS and pairwise- or reference-aligned LSU. Additionally, results of two-way ANOVA testing if Richness, Diversity (1-D), evenness $\left(E_{D}\right)$ and NMDS axes loading scores change with time, differ across treatments, or interact between the main effects. Lyman glacier forefront study included soils sampled across glacier forefront under plants with different mycorrhizal ecologies - Abies lasiocarpa, Luetkia pectinata, Phyllodoce empetriformis, Saxifraga ferruginea, and nonvegetated bare soil - referred here by genus. The stored biomass experiment included treatments of different biomass covering - No Plastic/No Tarp (NN), No Plastic/Tarp (NT), Plastic/No Tarp (PN), Plastic/Tarp (PT) - referred here by their abbreviation. Significant treatment effects are denoted in bold; upon a significant treatment effect, descriptions of community shifts are explained under the Change column. 
Figure 1
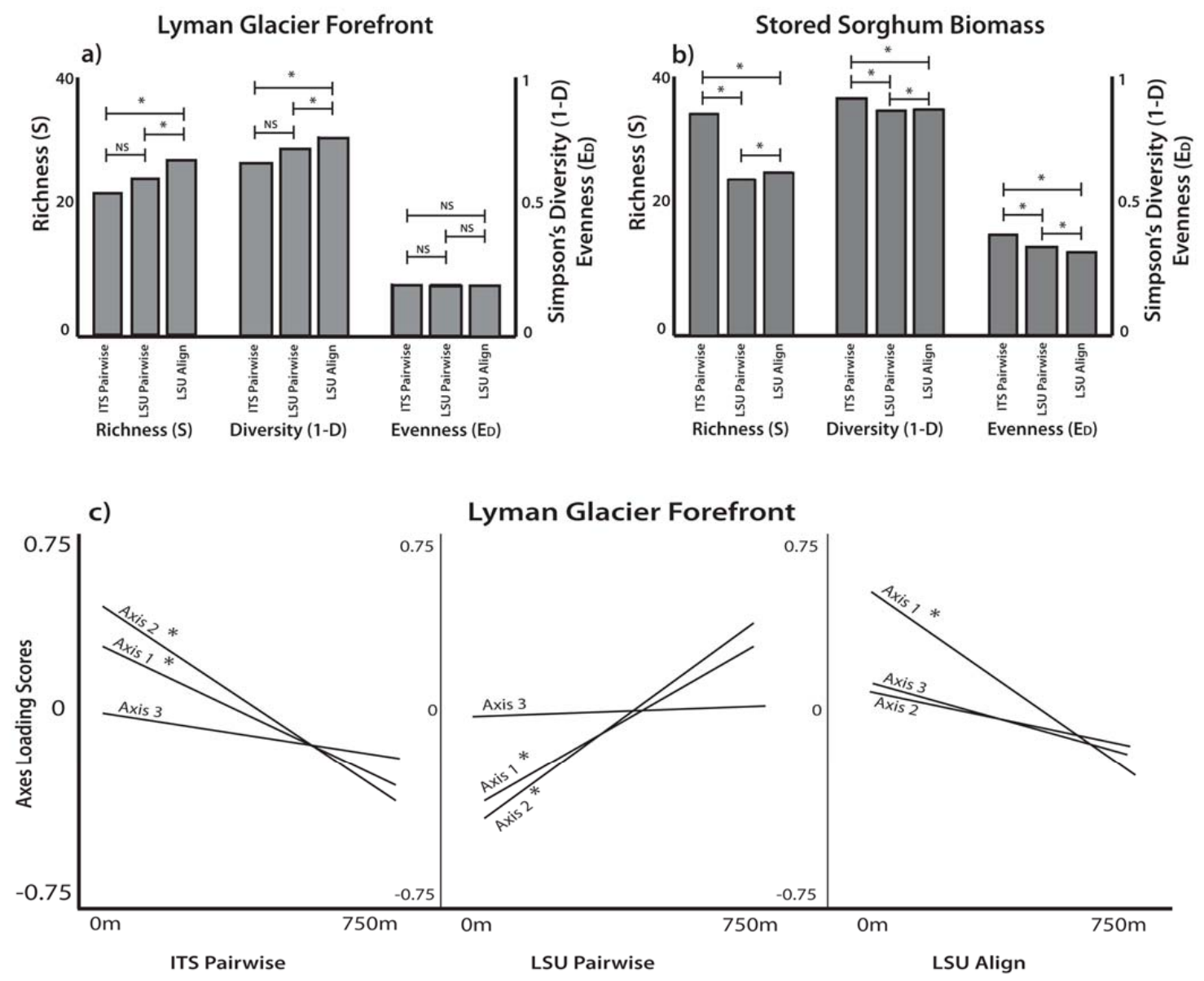

d)

Stored Sorghum Biomass

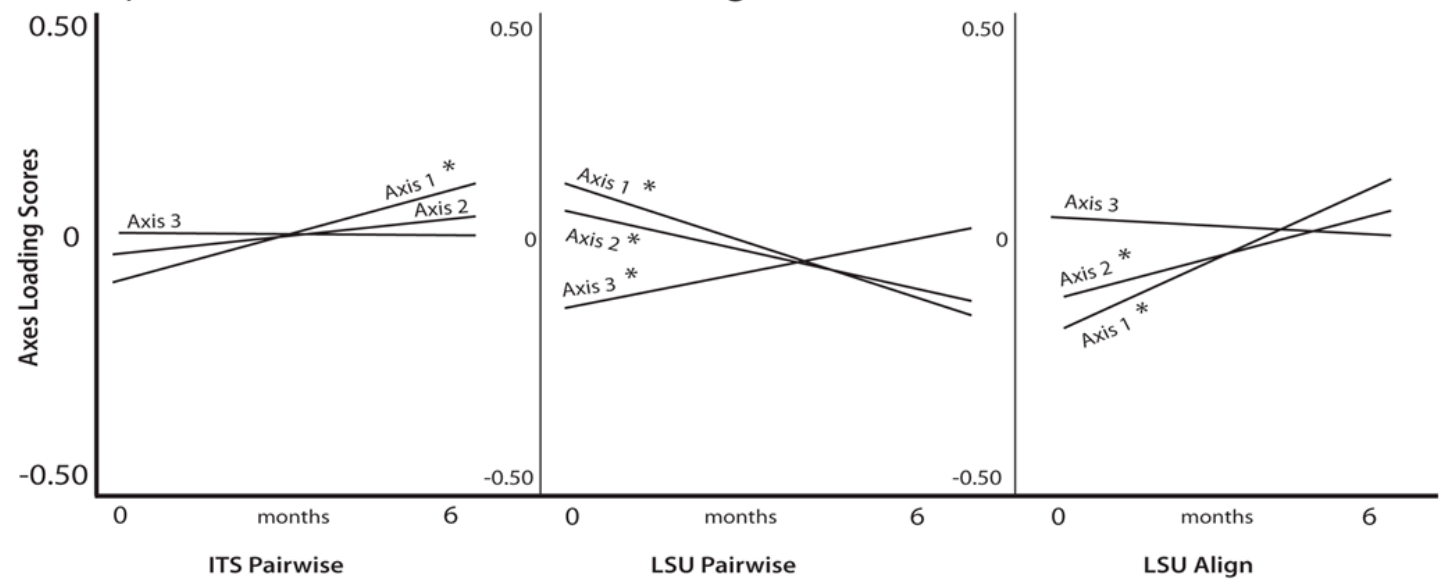




\section{Figure S1}
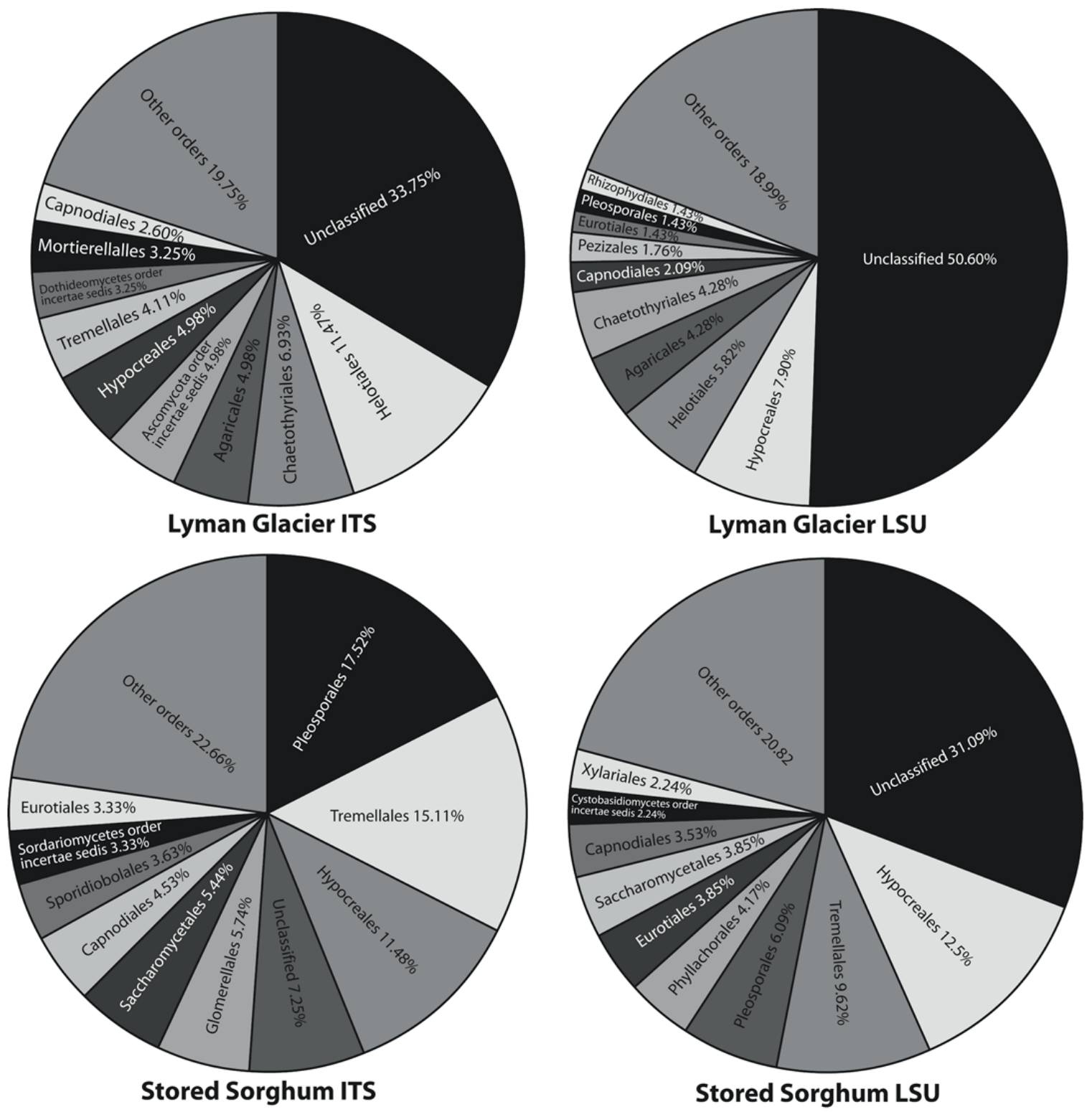

Table S1 
